\title{
Numerical Solution to the Multi-Term Time Fractional Diffusion Equation in a Finite Domain
}

\author{
Gongsheng Li*, Chunlong Sun, Xianzheng Jia, Dianhu Du \\ School of Sciences, Shandong University of Technology, Zibo, \\ Shandong 255049, China.
}

Received 16 September 2013; Accepted 21 May 2015

\begin{abstract}
This paper deals with numerical solution to the multi-term time fractional diffusion equation in a finite domain. An implicit finite difference scheme is established based on Caputo's definition to the fractional derivatives, and the upper and lower bounds to the spectral radius of the coefficient matrix of the difference scheme are estimated, with which the unconditional stability and convergence are proved. The numerical results demonstrate the effectiveness of the theoretical analysis, and the method and technique can also be applied to other kinds of time/space fractional diffusion equations.
\end{abstract}

AMS subject classifications: 35R11; 65M06; 65M12

Key words: Multi-term time fractional diffusion; finite difference scheme; spectral radius; stability and convergence; numerical simulation.

\section{Introduction}

The partial differential equations of fractional order have played an important role in modeling of the anomalous phenomena and in the theory of the complex systems during the last two decades, see, e.g., [1-4,8,9,20,24,25]. The so-called time-fractional diffusion equation that is obtained from the classical diffusion equation by replacing the first-order time derivative by a fractional derivative of order $\alpha$ with $0<\alpha<1$ has to be especially mentioned. On the other hand, by the attempts to describe some real processes with the equations of the fractional order, several researches confronted with the situation that the order $\alpha$ of the time-fractional derivative from the corresponding model equations did not remain constant and changed, say, in the interval from 0 to 1 , from 1 to 2 or even from 0 to 2 . To manage these phenomena, several approaches were suggested. One of them introduces the fractional derivatives of the variable order, i.e., the derivatives with the order that can change with the time or/and depending on the spatial coordinates $[5,6,16,22]$, and the other way is to employ the multi-term time

${ }^{*}$ Corresponding author. Email addresses: ligs@sdut.edu.cn (Gongsheng Li), sunchunlong527@163.com (Chunlong Sun), kathy1978@126.com (Xianzheng Jia), dudianhu@126.com (Dianhu Du) 
fractional differential equations. For $l>0, T>0$, the 1D multi-term time fractional diffusion equation in a finite homogeneous domain is given as

$$
\frac{\partial^{\alpha} u}{\partial t^{\alpha}}+\sum_{s=1}^{S} r_{s} \frac{\partial^{\beta_{s}} u}{\partial t^{\beta_{s}}}=D \frac{\partial^{2} u}{\partial x^{2}}+f(x, t), \quad 0<x<l, \quad 0<t<T,
$$

where $u=u(x, t)$ denotes the state variable at space point $x$ and time $t$, and $\alpha$ denotes the principal fractional order, and $\beta_{1}, \beta_{2}, \cdots, \beta_{S}$ are the multi-term fractional orders of the time derivatives, which satisfy the condition:

$$
0<\beta_{S}<\beta_{S-1}<\cdots<\beta_{1}<\alpha<1,
$$

and $r_{1}, \cdots, r_{S}$ are positive constants, and $D>0$ is the diffusion coefficient, $f(x, t)$ is a linear source term. All of the above time fractional derivatives are defined in the sense of Caputo, for example, the fractional derivative of the order $\beta \in(0,1)$ is given as

$$
\frac{\partial^{\beta} u}{\partial t^{\beta}}=\frac{1}{\Gamma(1-\beta)} \int_{0}^{t} \frac{\partial u(x, s)}{\partial s} \frac{d s}{(t-s)^{\beta}} .
$$

See, e.g., Podlubny [23] and Kilbas et al. [11] for the definition and properties of the Caputo's derivative.

There are still a few research works reported on the multi-term time fractional diffusion equations like Eq. (1.1). On theoretical analysis and analytical methods, we refer to Daftardar-Gejji et al. [7], Luchko [19] and Jiang et al. [10]. In [7], the multi-term time-fractional diffusion-wave equation with the constant coefficients was considered, and a solution of the corresponding IBV problem was represented in form of the Fourier series via the multivariate Mittag-Leffler function. In [19], a generalized multi-term time fractional diffusion equation with the variable coefficients was considered, and well-posedness of the corresponding IBV problem was proved with the help of maximum principle together with the construction of solution's representation using the Fourier method. In [10], analytical solutions of the 1D multi-term time fractional diffusion-wave/diffusion equations were obtained also using the Fourier's separating variables method. However, numerical solutions for the multi-term time fractional diffusion equations are of the same importance as the analytical solutions, especially when deal with concrete computations for real diffusion phenomena. Recently in [15], Liu et al. considered numerical solution to the multi-term time fractional wave-diffusion equation, and put forward a fractional predictor-corrector method by transforming the equation into a system of time-fractional differential equations, and they gave the convergence rate of the proposed algorithm without discussing the stability.

In this paper, we continue to deal with numerical methods for solving Eq. (1.1) with the initial condition

$$
u(x, 0)=u_{0}(x), \quad 0 \leq x \leq l ;
$$

and the homogeneous boundary condition

$$
u(0, t)=u(l, t)=0, \quad 0<t \leq T,
$$


where the initial function $u_{0}(x)$ and the source term $f(x, t)$ satisfy suitable conditions such that the initial boundary value problem (1.1), (1.4) and (1.5) has only one solution in suitable space. For example, if $u_{0}(x) \in C([0, l])$, and $f(x, t) \in C((0, l) \times(0, T))$, then there exists at most one classical solution $u(x, t) \in C([0, l] \times(0, T])$ for the above forward problem (see, e.g., $[17,18]$ ).

We give an implicit finite difference scheme for solving the forward problem (1.1), (1.4) and (1.5) numerically, and we are not only to prove the convergence but also to establish the unconditional stability for the difference scheme. It is noted that the unconditional stability and convergence of the difference scheme are proved in a simple way with the help of the estimation to the spectral radius of the coefficient matrix based on the discretization to the fractional derivations. Such method seems to be more effective as compared with those previous works (see, e.g., [13,14,21]), and we present several numerical examples to support the theoretical analysis.

The rest of the paper is organized as follows. In Section 2, the implicit finite difference scheme to the forward problem is put forward by discretization to the fractional derivations. In Section 3, unconditional stability and convergence of the difference scheme are proved by estimating the spectral radius of the coefficient matrix. In Section 4, numerical examples are presented to support the convergence analysis, and several concluding remarks are given in Section 5.

\section{The implicit finite difference scheme}

Firstly, discretizing the space domain by $x_{i}=i h(i=0,1, \cdots, M)$, and the time domain by $t_{n}=n \tau(n=0,1, \cdots, N)$, we have by definition (1.3)

$$
\begin{aligned}
& \frac{\partial^{\alpha} u}{\partial t^{\alpha}}\left(x_{i}, t_{n+1}\right) \\
& \quad=\frac{\tau^{-\alpha}}{\Gamma(2-\alpha)} \sum_{k=0}^{n}\left(u\left(x_{i}, t_{n+1-k}\right)-u\left(x_{i}, t_{n-k}\right)\right)\left[(k+1)^{1-\alpha}-k^{1-\alpha}\right]+O(\tau), \\
& \frac{\partial^{\beta_{s}} u}{\partial t^{\beta_{s}}}\left(x_{i}, t_{n+1}\right) \\
& \quad=\frac{\tau^{-\beta_{s}}}{\Gamma\left(2-\beta_{s}\right)} \sum_{k=0}^{n}\left(u\left(x_{i}, t_{n+1-k}\right)-u\left(x_{i}, t_{n-k}\right)\right)\left[(k+1)^{1-\beta_{s}}-k^{1-\beta_{s}}\right]+O(\tau),
\end{aligned}
$$

for $s=1,2, \cdots, S$, respectively, here $h=l / M$ is the space mesh step, and $\tau=T / N$ is the time mesh step.

Next, we discretize the integer-order derivative $\frac{\partial^{2} u}{\partial x^{2}}$ in Eq. (1.1) by utilizing general two-order center difference scheme, which is given by

$$
\frac{\partial^{2} u\left(x_{i}, t_{n+1}\right)}{\partial x^{2}}=\frac{u\left(x_{i+1}, t_{n+1}\right)-2 u\left(x_{i}, t_{n+1}\right)+u\left(x_{i-1}, t_{n+1}\right)}{h^{2}}+O\left(h^{2}\right) .
$$


Then denoting $u_{i}^{n}=u\left(x_{i}, t_{n}\right)$ and $f_{i}^{n}=f\left(x_{i}, t_{n}\right)$, and substituting (2.1)-(2.3) into Eq. (1.1), and multiplying by $\tau^{\alpha} \Gamma(2-\alpha)$, we have

$$
\begin{aligned}
& a \cdot\left(u_{i}^{n+1}-u_{i}^{n}\right)+a \sum_{k=1}^{n}\left(u_{i}^{n+1-k}-u_{i}^{n-k}\right) d_{k} \\
= & \frac{D \tau^{\alpha} \Gamma(2-\alpha)}{h^{2}}\left(u_{i-1}^{n+1}-2 u_{i}^{n+1}+u_{i+1}^{n+1}\right)+\tau^{\alpha} \Gamma(2-\alpha) f_{i}^{n+1}+R^{n+1},
\end{aligned}
$$

where

$$
\begin{aligned}
& a=1+\sum_{s=1}^{S} r_{s} \tau^{\alpha-\beta_{s}} \frac{\Gamma(2-\alpha)}{\Gamma\left(2-\beta_{s}\right)} \\
& d_{k}=\frac{1}{a}\left(\left[(k+1)^{1-\alpha}-k^{1-\alpha}\right]+\sum_{s=1}^{S} r_{s}\left[(k+1)^{1-\beta_{s}}-k^{1-\beta_{s}}\right] \tau^{\alpha-\beta_{s}} \frac{\Gamma(2-\alpha)}{\Gamma\left(2-\beta_{s}\right)}\right),
\end{aligned}
$$

for $k=0,1, \cdots, n$. Moreover, it follows from (2.1)-(2.3) that the remainder in (2.4) satisfies

$$
R^{n+1}=\tau^{\alpha} \Gamma(2-\alpha) O\left(h^{2}+\tau\right)=O\left(h^{2} \tau^{\alpha}+\tau^{\alpha+1}\right) .
$$

Let

$$
p=\frac{D \tau^{\alpha} \Gamma(2-\alpha)}{h^{2} a} .
$$

Ignoring the remainder term in (2.4) and dividing both sides of (2.4) by $a$, we have

$$
\begin{aligned}
& -p u_{i-1}^{n+1}+(1+2 p) u_{i}^{n+1}-p u_{i+1}^{n+1} \\
= & u_{i}^{n}-\sum_{k=1}^{n} d_{k}\left(u_{i}^{n+1-k}-u_{i}^{n-k}\right)+\frac{1}{a} \tau^{\alpha} \Gamma(2-\alpha) f_{i}^{n+1} .
\end{aligned}
$$

The initial boundary conditions are discretized as

$$
u_{i}^{0}=u_{0}\left(x_{i}\right) ; \quad u_{0}^{n}=0, \quad u_{M}^{n}=0 .
$$

Let

$$
\begin{aligned}
& U^{n}=\left(u_{1}^{n}, \cdots, u_{M-1}^{n}\right)^{\prime}, \quad U^{0}=\left(u_{1}^{0}, \cdots, u_{M-1}^{0}\right)^{\prime}, \quad f^{n}=\left(f_{1}^{n}, \cdots, f_{M-1}^{n}\right)^{\prime} ; \\
& c_{k}=d_{k-1}-d_{k}, \quad k=1, \cdots, n,
\end{aligned}
$$

where $d_{k}(k=0,1, \cdots, n)$ is defined by (2.6); and $B=\left(b_{i j}\right)_{(M-1) \times(M-1)}$, where $b_{i j}=0$ for $1 \leq i, j \leq M-1$ except

$$
b_{i j}= \begin{cases}-p, & j=i+1, \\ 1+2 p, & j=i, \\ -p, & j=i-1 .\end{cases}
$$


Thus, we have the implicit difference scheme in the matrix form given by

$$
\begin{aligned}
& B U^{1}=U^{0}+\frac{1}{a} \tau^{\alpha} \Gamma(2-\alpha) f^{1} \\
& B U^{n+1}=c_{1} U^{n}+c_{2} U^{n-1}+\cdots+c_{n} U^{1}+d_{n} U^{0}+\frac{1}{a} \tau^{\alpha} \Gamma(2-\alpha) f^{n+1} .
\end{aligned}
$$

Obviously, the coefficient matrix $B$ is symmetric, and strictly diagonally dominant, and the difference equation (2.11) has only one solution. In the next subsection, we will prove its stability and convergence with the help of analysis to the coefficient matrix.

\section{The stability and convergence}

Firstly, we give several lemmas.

Lemma 3.1. For $k=1, \cdots, n$, and $0<\alpha<1$, there is

$$
2 k^{\alpha}-(k-1)^{\alpha}-(k+1)^{\alpha}>0 .
$$

Proof. Let $f(x)=(x+1)^{\alpha}-x^{\alpha}$, for $x>0$ and $0<\alpha<1$. It is easy to testify that $f^{\prime}(x)<0$, and then the function $f(x)$ is strictly decreasing for $x>0$, and so there is $f(k)<f(k-1)$ for $k>1$. This proves (3.1).

Lemma 3.2. For $0<\alpha, \beta_{s}<1(s=1, \cdots, S)$, and $c_{k}$, $d_{k}$ given by (2.9) and (2.6) respectively. Then $c_{k}>0(k=1, \cdots, n)$, and

$$
\sum_{k=1}^{n} c_{k}+d_{n}=1 .
$$

Proof. By (2.6) and (2.9), we have

$$
\begin{gathered}
c_{k}=d_{k-1}-d_{k}=k^{1-\alpha}-(k-1)^{\alpha}+\sum_{s=1}^{S} r_{s} \tau^{\alpha-\beta} \frac{\Gamma(2-\alpha)}{\Gamma(2-\beta)}\left[k^{1-\beta}-(k-1)^{\beta}\right] \\
\quad-(k+1)^{1-\alpha}+k^{\alpha}-\sum_{s=1}^{S} r_{s} \tau^{\alpha-\beta} \frac{\Gamma(2-\alpha)}{\Gamma(2-\beta)}\left[(k+1)^{1-\beta}-k^{\beta}\right], \\
=2 k^{1-\alpha}-(k-1)^{1-\alpha}-(k+1)^{1-\alpha} \\
\quad+\sum_{s=1}^{S} r_{s} \tau^{\alpha-\beta} \frac{\Gamma(2-\alpha)}{\Gamma(2-\beta)}\left[2 k^{1-\beta}-(k-1)^{1-\beta}-(k+1)^{1-\beta}\right] .
\end{gathered}
$$

It follows from (3.1) that $c_{k}>0$ for $k=1, \cdots, n$. In addition, noting that $c_{k}=$ $d_{k-1}-d_{k}$, and $d_{0}=1$, we can easily verify (3.2).

Next, noting to definitions of the elements $b_{i j}$ of the matrix $B$ and $p$ given by (2.7), we have 
Lemma 3.3. If $D, r_{s}>0(s=1, \cdots, S)$, and $0<\beta_{S}<\beta_{S-1}<\cdots<\beta_{1}<\alpha<1$, then $p>0$ and

$$
\begin{aligned}
& b_{i i}=1+\sum_{j=1, j \neq i}^{M-1}\left|b_{i j}\right|, \quad 1 \leq i \leq M-1 \\
& \sum_{j=1, j \neq i}^{M-1} b_{i j}<0, \quad 1 \leq i \leq M-1 .
\end{aligned}
$$

Now, we give an estimate of the spectral radius of the coefficient matrix $B$ defined by $(2.10)$.

Theorem 3.1. Under the conditions of Lemma 3.3, and note that $B$ is a symmetry matrix, we have

$$
\begin{aligned}
& 1 \leq \rho(B)=\|B\|_{2} \leq 2\|b\|_{\infty}-1, \\
& \frac{1}{2\|b\|_{\infty}-1} \leq \rho\left(B^{-1}\right)=\left\|B^{-1}\right\|_{2} \leq 1,
\end{aligned}
$$

where $\|b\|_{\infty}=\max _{1 \leq i \leq M-1}\left\{b_{i i}\right\}$, and $\rho(B)$ is the spectral radius of $B$, and $\|B\|_{2}$ denotes the 2-norm of the matrix $B$.

Proof. Suppose that

$$
B \Theta=\lambda \Theta,
$$

where $\lambda$ denotes an eigenvalue of matrix $B$, and $\Theta=\left(\theta_{1}, \cdots, \theta_{M-1}\right) \neq 0$ is the corresponding eigenvector. Without loss of generality, setting $\left|\theta_{k}\right|=\max _{1 \leq i \leq M-1}\left|\theta_{i}\right|$, and considering the $k$-th equation of $B \Theta=\lambda \Theta$, we have

$$
\sum_{j=1}^{M-1} b_{k j} \theta_{j}=\lambda \theta_{k},
$$

which gives

$$
\lambda-b_{k k}=\sum_{j=1, j \neq k}^{M-1} b_{k j} \theta_{j} / \theta_{k} .
$$

Using Lemma 3.3 and noting $\left|\theta_{j} / \theta_{k}\right| \leq 1$, we obtain

$$
\left|\lambda-b_{k k}\right| \leq \sum_{j=1, j \neq k}^{M-1}\left|b_{k j}\right|=b_{k k}-1 .
$$

Consequently,

$$
1 \leq|\lambda| \leq 2 b_{k k}-1, \quad k=1, \cdots, M-1
$$


which implies the assertions (3.4) and (3.5) are both valid.

Based on the above analysis, and with a similar method as used in [12], we can prove the unconditional stability and convergence for the implicit finite difference scheme (2.11). By theorem 3.1, we will prove the stability and convergence of the difference scheme using the 2-norm of the matrix $B$ or vector $E$, and for convenience of writing we still denote them as $\|B\|$ and $\|E\|$ in the following statements.

Theorem 3.2. The implicit difference scheme defined by (2.11) is unconditionally stable.

Proof. By the linear difference scheme (2.11), we can easily get

$$
\left\{\begin{array}{l}
B E^{1}=E^{0}, \quad E^{0}=\widetilde{U}^{0}-U^{0} \\
B E^{n+1}=c_{1} E^{n}+\cdots+c_{n} E^{1}+d_{n} E^{0},
\end{array}\right.
$$

where $\widetilde{U}^{0}$ denotes the initial function with noises, $E^{n}=\widetilde{U}^{n}-U^{n}$ denotes the solutions difference at the $n$-th level, and $n=0,1, \cdots$.

Obviously, by (3.6) and Theorem 3.1 we have

$$
\left\|E^{1}\right\| \leq\left\|B^{-1}\right\|\left\|E^{0}\right\| \leq\left\|E^{0}\right\| .
$$

Suppose that there is $\left\|E^{k}\right\| \leq\left\|E^{0}\right\|$ for $k \leq n$, then also utilizing Theorem 3.1 and (3.6), we have

$$
\begin{aligned}
\left\|E^{n+1}\right\| & \leq\left\|B^{-1}\right\|\left\|c_{1} E^{n}+\cdots+c_{n} E^{1}+d_{n} E^{0}\right\| \\
& \leq c_{1}\left\|E^{n}\right\|+\cdots+c_{n}\left\|E^{1}\right\|+d_{n}\left\|E^{0}\right\| .
\end{aligned}
$$

Using the assumptions, $\left\|E^{k}\right\| \leq\left\|E^{0}\right\|(k=1, \cdots, n)$, we can get

$$
\left\|E^{n+1}\right\| \leq\left(c_{1}+\cdots+c_{n}+d_{n}\right)\left\|E^{0}\right\| .
$$

It follows from Lemma 3.2 that $\left\|E^{n+1}\right\| \leq\left\|E^{0}\right\|$, which implies the unconditional stability of the difference scheme (2.11).

Denote $e_{i}^{n}=u\left(x_{i}, t_{n}\right)-u_{i}^{n}(i=1, \cdots, M-1, n=1, \cdots, N-1)$, where $u\left(x_{i}, t_{n}\right)$ be the exact solution of the forward problem (1.1) with (1.4)-(1.5) at mesh point $\left(x_{i}, t_{n}\right)$, and $u_{i}^{n}$ is the solution of the difference scheme (2.11) also at $\left(x_{i}, t_{n}\right)$, and $e^{n}=\left(e_{1}^{n}, e_{2}^{n}, \cdots, e_{M-1}^{n}\right)$. Noting that $e_{i}^{0}=u\left(x_{i}, 0\right)-u_{i}^{0}=0$, there is $e^{0}=0$. We give the convergence result.

Theorem 3.3. The difference solution of (2.11) is convergent to the exact solution of the forward problem with the convergence order of $O\left(h^{2}+\tau\right)$ as $h, \tau \rightarrow 0$ for any finite time $T<\infty$, and there is

$$
\left\|e_{n}\right\| \leq \frac{M a}{1-\alpha+\sum_{s=1}^{S} r_{s}\left(1-\beta_{s}\right) T^{\alpha-\beta_{s}} \frac{\Gamma(2-\alpha)}{\Gamma\left(2-\beta_{s}\right)}} T^{\alpha}\left(h^{2}+\tau\right),
$$

where $a \geq 1$ is defined by (2.5). 
Proof. Also by the linear difference system (2.11), we have

$$
\left\{\begin{array}{l}
B e^{1}=R^{1} \\
B e^{n+1}=c_{1} e^{n}+\cdots+c_{n} e^{1}+R^{n+1}
\end{array}\right.
$$

where $R^{n}=\left(R_{1}^{n}, R_{2}^{n}, \cdots, R_{M-1}^{n}\right)$ denotes the truncated term in the solutions' approximation for $n=1,2, \cdots$. By the expressions (2.1)-(2.4), there is a constant $M>0$ such that

$$
\left\|R^{n}\right\| \leq M \tau^{\alpha}\left(h^{2}+\tau\right), \quad n=1,2, \cdots
$$

with which and also using Theorem 3.1 and Lemma 3.2 we can prove the convergence.

Actually, by (3.9) and using Theorem 3.1, we have

$$
\left\|e^{1}\right\| \leq\left\|B^{-1}\right\|\left\|R^{1}\right\| \leq\left\|R^{1}\right\| \leq M \tau^{\alpha}\left(h^{2}+\tau\right) .
$$

Moreover, we can prove by induction that

$$
\left\|e^{n+1}\right\| \leq \frac{1}{d_{n} n^{\alpha}} M T^{\alpha}\left(h^{2}+\tau\right)
$$

where $d_{n}$ is given by (2.6). In fact, we have for $n=1$

$$
\left\|e^{2}\right\| \leq c_{1}\left\|e^{1}\right\|+\left\|R^{2}\right\|=c_{1}\left\|e^{1}\right\|+d_{1}\left\|R^{2}\right\| / d_{1} .
$$

Using Lemma 3.2, (3.11) and (3.10), we have

$$
\left\|e^{2}\right\| \leq \frac{1}{d_{1}}\left(c_{1}\left\|e^{1}\right\|+d_{1}\left\|R^{2}\right\|\right) \leq \frac{1}{d_{1}}\left(c_{1}+d_{1}\right) M \tau^{\alpha}\left(h^{2}+\tau\right)=\frac{1}{d_{1}} M \tau^{\alpha}\left(h^{2}+\tau\right) .
$$

Similarly, we can get

$$
\left\|e^{n+1}\right\| \leq \frac{1}{d_{n}}\left(\sum_{k=1}^{n} c_{k}+d_{n}\right) M \tau^{\alpha}\left(h^{2}+\tau\right) .
$$

Again using Lemma 3.2 and noting $n \tau \leq T$, we get the assertion (3.12). On the other hand, we have (see Appendix A)

$$
\lim _{n \rightarrow \infty} \frac{1}{d_{n} n^{\alpha}}=\frac{a}{1-\alpha+\sum_{s=1}^{S} r_{s}\left(1-\beta_{s}\right) T^{\alpha-\beta_{s}} \Gamma(2-\alpha) / \Gamma\left(2-\beta_{s}\right)},
$$

where $a$ is given by (2.5). Then by (3.12) we deduce that for sufficiently large $n$ and for finite time $T$, the assertion of the theorem is valid.

Remark 3.1. By (3.8) we can see that the time step $\tau$ is more important than the space step $h$, and it should be sufficiently small in order to get more accurate numerical solutions. In addition, we find that if $\beta_{s}=\alpha$ for all $s=1,2, \cdots, S$, then we have also by (3.8)

$$
\left\|e_{n}\right\| \leq \frac{M}{1-\alpha} T^{\alpha}\left(h^{2}+\tau\right)
$$

which is in accordance with the assertion for one-term time-fractional diffusion equation given in [12]. 


\section{Numerical examples}

\subsection{Solution to the homogeneous equation}

Consider the solution to the homogeneous equation (1.1) with two-term and threeterm time fractional derivatives, i.e., we are concerned with the two-term time fractional diffusion equation

$$
\frac{\partial^{\alpha} u}{\partial t^{\alpha}}+r_{0} \frac{\partial^{\beta} u}{\partial t^{\beta}}-D \frac{\partial^{2} u}{\partial x^{2}}=0
$$

and the three-term time fractional diffusion equation

$$
\frac{\partial^{\alpha} u}{\partial t^{\alpha}}+r_{1} \frac{\partial^{\beta} u}{\partial t^{\beta}}+r_{2} \frac{\partial^{\gamma} u}{\partial t^{\gamma}}-D \frac{\partial^{2} u}{\partial x^{2}}=0
$$

respectively, for which we can get their analytical solutions by using the method of separating variables and Laplace transform (see Appendix B).

Example 4.1. We firstly consider the two-term time fractional diffusion equation (4.1), where $\alpha$ and $\beta$ are the factional orders satisfying $0<\beta \leq \alpha<1$. Setting $l=\pi, T=1$, and $D=1, r_{0}=0.5$, and the initial function $u_{0}(x)=\sin (x)$, then the analytical solution is given as

$$
\begin{aligned}
u(x, t)=\sum_{k=0}^{\infty} & (-1)^{k} \frac{D^{k}}{k !} t^{\alpha k}\left(E_{\alpha-\beta, \beta k+1}^{(k)}\left(-r_{0} t^{\alpha-\beta}\right)\right. \\
& \left.+r_{0} t^{\alpha-\beta} E_{\alpha-\beta, \alpha-\beta+\beta k+1}^{(k)}\left(-r_{0} t^{\alpha-\beta}\right)\right) \sin (x) .
\end{aligned}
$$

On the concrete computations, noting that

$$
E_{\alpha, \beta}^{(k)}=\sum_{j=0}^{\infty} \frac{(k+j) ! z^{j}}{j ! \Gamma(\alpha(k+j)+\beta)},
$$

there are

$$
\begin{aligned}
& E_{\alpha-\beta, \beta k+1}^{(k)}\left(-r_{0} t^{\alpha-\beta}\right)=\sum_{j=0}^{\infty} \frac{(k+j) !\left(-r_{0} t^{\alpha-\beta}\right)^{j}}{j ! \Gamma((\alpha-\beta)(k+j)+\beta k+1)} \\
& E_{\alpha-\beta, \alpha-\beta+\beta k+1}^{(k)}\left(-r_{0} t^{\alpha-\beta}\right)=\sum_{j=0}^{\infty} \frac{(k+j) !\left(-r_{0} t^{\alpha-\beta}\right)^{j}}{j ! \Gamma((\alpha-\beta)(k+j)+\alpha-\beta+\beta k+1)},
\end{aligned}
$$

respectively. Then the analytical solution is approximated by

$$
\begin{aligned}
u(x, t) \approx & \sum_{k=0}^{K}(-1)^{k} \frac{D^{k}}{k !} t^{\alpha k}\left(\sum_{j=0}^{J} \frac{(k+j) !\left(-r_{0} t^{\alpha-\beta}\right)^{j}}{j ! \Gamma((\alpha-\beta)(k+j)+\beta k+1)}\right. \\
& \left.+r_{0} t^{\alpha-\beta} \sum_{j=0}^{J} \frac{(k+j) !\left(-r_{0} t^{\alpha-\beta}\right)^{j}}{j ! \Gamma((\alpha-\beta)(k+j)+\alpha-\beta+\beta k+1)}\right) \sin (x) .
\end{aligned}
$$


Without loss of generality, we take $\alpha=0.6$ and $\beta=0.4$ as example, and choose $K=J=50$ in the formula (4.4) to get the analytical solution. The relative errors in the solutions at $t=0.5$ with different space and time steps are listed in Table 1 and Table 2, where $h, \tau$ denote the space and time steps respectively, and the relative error is given by

$$
\operatorname{Err}=\frac{\left\|u(x, 0.5)-u^{*}(x, 0.5)\right\|_{2}}{\|u(x, 0.5)\|_{2}},
$$

and $u(x, 0.5)$ is the analytical solution approximated by $(4.4)$, and $u^{*}(x, 0.5)$ is the difference solution worked out by (2.11).

Table 1: The solutions errors with time steps for $h=\pi / 100$ in Example 4.1.

\begin{tabular}{|cccccc|}
\hline$\tau$ & $1 / 100$ & $1 / 200$ & $1 / 300$ & $1 / 500$ & $1 / 1000$ \\
\hline Err & $2.3016 \mathrm{e}-3$ & $1.1524 \mathrm{e}-3$ & $7.7479 \mathrm{e}-4$ & $4.7529 \mathrm{e}-4$ & $2.5268 \mathrm{e}-4$ \\
\hline
\end{tabular}

Table 2: The solutions errors with space steps for $\tau=1 / 100$ in Example 4.1.

\begin{tabular}{|cccccc|}
\hline$h$ & $\pi / 100$ & $\pi / 200$ & $\pi / 300$ & $\pi / 500$ & $\pi / 1000$ \\
\hline Err & $2.3016 \mathrm{e}-3$ & $2.2769 \mathrm{e}-3$ & $2.2724 \mathrm{e}-3$ & $2.2700 \mathrm{e}-3$ & $2.2690 \mathrm{e}-3$ \\
\hline
\end{tabular}

From Tables 1 and 2, we find that the solutions errors become small as the grid steps small, but the convergent rate is slow for the space and time steps varied with the same scale. Noting to Theorem 3.3, we recompute the numerical solutions also for $\alpha=0.6$ and $\beta=0.4$ but with the grid scale of $\tau=h^{2} / \pi^{2}$, the computational results are listed in Table 3.

Table 3: The solutions errors with time and space steps in Example 4.1.

\begin{tabular}{|cccc|}
\hline$h$ & $\tau=h^{2} / \pi^{2}$ & Err & Rat \\
\hline$\pi / 4$ & $1 / 16$ & $3.52456 \mathrm{e}-2$ & \\
$\pi / 10$ & $1 / 100$ & $5.55438 \mathrm{e}-3$ & 6.3456 \\
$\pi / 20$ & $1 / 400$ & $1.37756 \mathrm{e}-3$ & 4.0320 \\
$\pi / 40$ & $1 / 1600$ & $3.42765 \mathrm{e}-4$ & 4.0190 \\
$\pi / 80$ & $1 / 6400$ & $8.54593 \mathrm{e}-5$ & 4.0109 \\
\hline
\end{tabular}

By Table 3 we can see that the solutions errors approach to zero with almost the same rate as the steps become small in the case of $\tau=h^{2} / \pi^{2}$, and there exists a constant $M>0$ such that

$$
\operatorname{Err} \leq M\left(h^{2}+\tau\right), \quad h, \tau \rightarrow 0
$$

which is just in accordance with the convergence analysis given in Theorem 3.3.

Moreover, choosing $h=\pi / 40$ and $\tau=h^{2} / \pi^{2}=1 / 1600$, the relative errors in the solutions at $t=0.5$ with different fractional orders are listed in Table 4 , and the analytical solution and the difference solution at $t=0.5$ for $\alpha=0.6$ and $\beta=0.4$ are plotted in Fig. 1, respectively. 


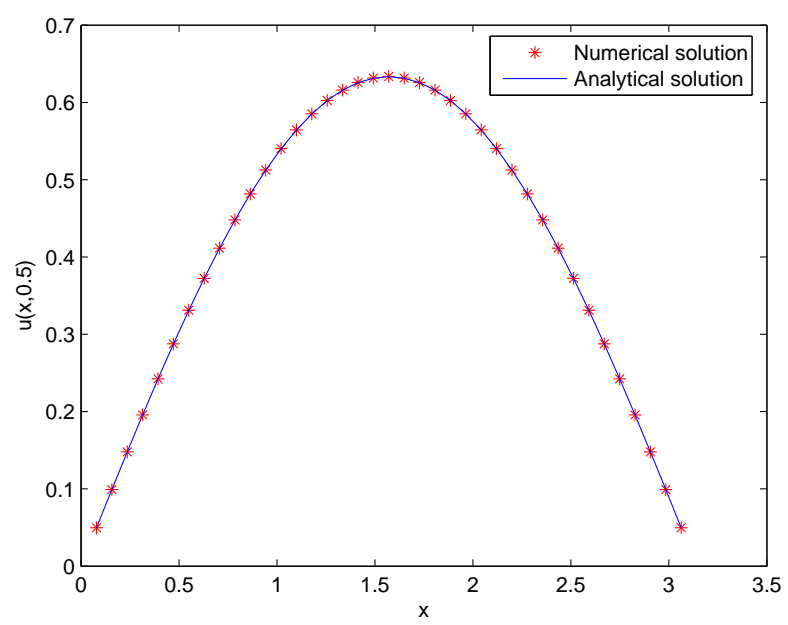

Figure 1: The analytical and numerical solutions for $\alpha=0.6, \beta=0.4$ in Example 4.1.

Table 4: The solutions errors with different fractional orders in Example 4.1.

\begin{tabular}{|ccc|}
\hline$\alpha$ & $\beta$ & Err \\
\hline 0.9 & 0.1 & $4.16195 \mathrm{e}-4$ \\
\hline 0.8 & 0.2 & $3.91113 \mathrm{e}-4$ \\
\hline 0.7 & 0.3 & $3.62738 \mathrm{e}-4$ \\
\hline 0.6 & 0.4 & $3.42765 \mathrm{e}-4$ \\
\hline 0.5 & 0.5 & $3.31074 \mathrm{e}-4$ \\
\hline
\end{tabular}

By the above computations, we find that the fractional orders have little influences on solving the forward problem, and the difference solution is convergent to the analytical solution as the mesh becoming fine. It seems to be more significant to the numerical solutions for reducing the time step than that of the space step, and the numerical solution converges to the analytical solution perfectly if choosing $\tau=h^{2} / \pi^{2}$ in this example.

Example 4.2. Consider the three-term time fractional diffusion equation (4.2), where $\alpha, \beta$ and $\gamma$ are the factional orders satisfying $0<\gamma \leq \beta \leq \alpha<1$. We also set $l=\pi, T=1$, and $D=1$, and the initial function $u_{0}(x)=\sin (x)$. The numerical solution is worked out by the difference scheme (2.11), and the analytical solution in this case is expressed by the following formula (also see Appendix B)

$$
\begin{aligned}
u(x, t)=\sum_{m=0}^{\infty} & (-1)^{m} \sum_{k=0}^{m} \frac{r_{2}^{k}}{k !(m-k) !} t^{\alpha m-k \gamma}\left(E_{\alpha-\beta, 1+m \beta-k \gamma}^{(m)}\left(-r_{1} t^{\alpha-\beta}\right)\right. \\
& +r_{1} t^{\alpha-\beta} E_{\alpha-\beta, 1+m \beta-k \gamma+\alpha-\beta}^{(m)}\left(-r_{1} t^{\alpha-\beta}\right) \\
& \left.+r_{2} t^{\alpha-\gamma} E_{\alpha-\beta, 1+m \beta-k \gamma+\alpha-\gamma}^{(m)}\left(-r_{1} t^{\alpha-\beta}\right)\right) \sin (x) .
\end{aligned}
$$

We choose the fractional orders $\alpha=0.8, \beta=0.5$ and $\gamma=0.3$, and the coefficients 


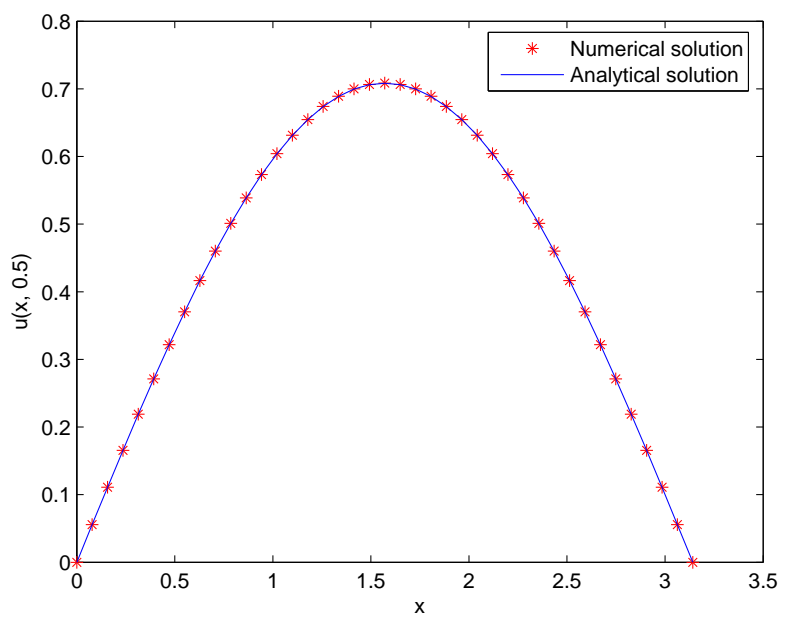

Figure 2: The analytical and numerical solutions for $\alpha=0.8, \beta=0.5, \gamma=0.3$ in Example 4.2.

$r_{1}=r_{2}=0.5$. Like done in Example 4.1, the relative errors in the solutions at $t=$ 0.5 with different space and time steps are listed in Tables 5-7 respectively, and the relative error is also given by (4.5). Moreover, the analytical solution and the difference solution at $t=0.5$ for $\alpha=0.8$ and $\beta=0.5, \gamma=0.3$ are plotted in Fig. 2 .

Table 5: The solutions errors with time steps for $h=\pi / 100$ in Example 4.2.

\begin{tabular}{|cccccc|}
\hline$\tau$ & $1 / 100$ & $1 / 200$ & $1 / 300$ & $1 / 500$ & $1 / 1000$ \\
\hline Err & $2.1402 \mathrm{e}-3$ & $1.0773 \mathrm{e}-3$ & $7.2490 \mathrm{e}-4$ & $4.04403 \mathrm{e}-4$ & $2.3433 \mathrm{e}-4$ \\
\hline
\end{tabular}

Table 6: The solutions errors with space steps for $\tau=1 / 100$ in Example 4.2.

\begin{tabular}{|cccccc|}
\hline$h$ & $\pi / 100$ & $\pi / 200$ & $\pi / 300$ & $\pi / 500$ & $\pi / 1000$ \\
\hline Err & $2.1402 \mathrm{e}-3$ & $2.1206 \mathrm{e}-3$ & $2.1169 \mathrm{e}-3$ & $2.1151 \mathrm{e}-3$ & $2.1143 \mathrm{e}-3$ \\
\hline
\end{tabular}

Table 7: The solutions errors with time and space steps in Example 4.2.

\begin{tabular}{|cccc|}
\hline$h$ & $\tau=h^{2} / \pi^{2}$ & Err & Rat \\
\hline$\pi / 4$ & $1 / 16$ & $2.92199 \mathrm{e}-2$ & \\
$\pi / 10$ & $1 / 100$ & $4.73759 \mathrm{e}-3$ & 6.1677 \\
$\pi / 20$ & $1 / 400$ & $1.18154 \mathrm{e}-3$ & 4.0097 \\
$\pi / 40$ & $1 / 1600$ & $2.94488 \mathrm{e}-4$ & 4.0122 \\
$\pi / 80$ & $1 / 6400$ & $7.34376 \mathrm{e}-5$ & 4.0100 \\
\hline
\end{tabular}

From Tables 5-7, we can see that the solutions errors have the same trends as observed in Tables 1-3, respectively, and the difference solution is also in good accordance with the analytical solution in the case of $\tau=h^{2} / \pi^{2}$. 


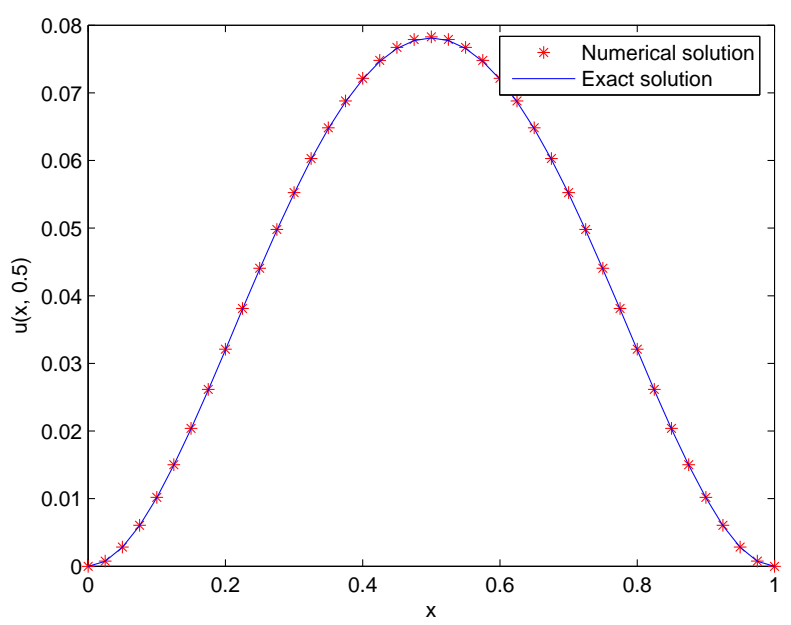

Figure 3: The numerical and exact solutions for $\alpha=0.7, \beta=0.6, \gamma=0.4$ in Example 4.3.

\subsection{Solution to the inhomogeneous equation}

In this subsection, we consider the inhomogeneous equation with three-term time fractional derivatives in the unit domain given as

$$
\frac{\partial^{\alpha} u}{\partial t^{\alpha}}+r_{1} \frac{\partial^{\beta} u}{\partial t^{\beta}}+r_{2} \frac{\partial^{\gamma} u}{\partial t^{\gamma}}-D \frac{\partial^{2} u}{\partial x^{2}}=f(x, t), \quad 0<x<1,0<t<1 .
$$

Example 4.3. We set the exact solution be

$$
u(x, t)=x^{2}(1-x)^{2}\left(1+t^{2}\right),
$$

in this example, and the initial function $u_{0}(x)=x^{2}(1-x)^{2}$. Noting to $\frac{\partial^{\beta}\left(t^{2}\right)}{\partial t^{\beta}}=\frac{2 t^{2-\beta}}{\Gamma(3-\beta)}$, we get the nonzero source term

$$
\begin{gathered}
f(x, t)=2 x^{2}(1-x)^{2}\left(\frac{t^{2-\alpha}}{\Gamma(3-\alpha)}+r_{1} \frac{t^{2-\beta}}{\Gamma(3-\beta)}+r_{2} \frac{t^{2-\gamma}}{\Gamma(3-\gamma)}\right) \\
-2 D\left(6 x^{2}-6 x+1\right)\left(1+t^{2}\right) .
\end{gathered}
$$

For other parameters in the model, we take $D=1$ and $r_{1}=1, r_{2}=0.5$, and we choose the fractional orders $\alpha=0.7, \beta=0.6$ and $\gamma=0.4$ in this example, and we only investigate the solutions errors at $t=0.5$ in the case of $\tau=h^{2}$. The computational results are listed in Table 8 , and the numerical solution with the exact solution are plotted in Fig. 3, respectively.

From Table 8, we can see that the solutions errors have the completely same trends as observed in Table 3 and Table 7, and all of them effectively support the convergence result given in Theorem 3.3. In the follows, we give an example in which the exact solution of the forward problem is related with the fractional orders. 
Table 8: The solutions errors with time and space steps in Example 4.3.

\begin{tabular}{|cccc|}
\hline$h$ & $\tau=h^{2}$ & Err & Rat \\
\hline $1 / 4$ & $1 / 16$ & $2.18714 \mathrm{e}-1$ & \\
$1 / 10$ & $1 / 100$ & $3.56113 \mathrm{e}-2$ & 6.1417 \\
$1 / 20$ & $1 / 400$ & $8.90798 \mathrm{e}-3$ & 3.9977 \\
$1 / 40$ & $1 / 1600$ & $2.22580 \mathrm{e}-3$ & 4.0021 \\
$1 / 80$ & $1 / 6400$ & $5.56142 \mathrm{e}-4$ & 4.0022 \\
\hline
\end{tabular}

Example 4.4. Also considering the inhomogeneous diffusion equation with three-term time fractional derivatives in the unit domain $(l=1)$, and we here set the exact solution be

$$
u(x, t)=x^{1+\alpha}(1-x)\left(1+t^{\beta+\gamma}\right),
$$

and the initial function $u_{0}(x)=x^{1+\alpha}(1-x)$. Then noting that

$$
\frac{\partial^{\beta}\left(t^{n}\right)}{\partial t^{\beta}}=\frac{\Gamma(n+1)}{\Gamma(n+1-\beta)} t^{n-\beta},
$$

the nonzero source term in this case is

$$
\begin{gathered}
f(x, t)=x^{1+\alpha}(1-x) \Gamma(\beta+\gamma+1)\left(\frac{t^{\beta+\gamma-\alpha}}{\Gamma(\beta+\gamma+1-\alpha)}+r_{1} \frac{t^{\gamma}}{\Gamma(\gamma+1)}+r_{2} \frac{t^{\beta}}{\Gamma(\beta+1)}\right) \\
-D(1+\alpha) x^{\alpha-1}[\alpha-(2+\alpha) x]\left(1+t^{\beta+\gamma}\right) .
\end{gathered}
$$

We also take $D=1$, and the fractional orders $\alpha=0.8, \beta=0.7$ and $\gamma=0.6$, and $r_{1}=0.5, r_{2}=1.0$ in this example. The solutions errors at $t=1$ are listed in Table 9, and the exact and numerical solutions are plotted in Fig. 4, respectively.

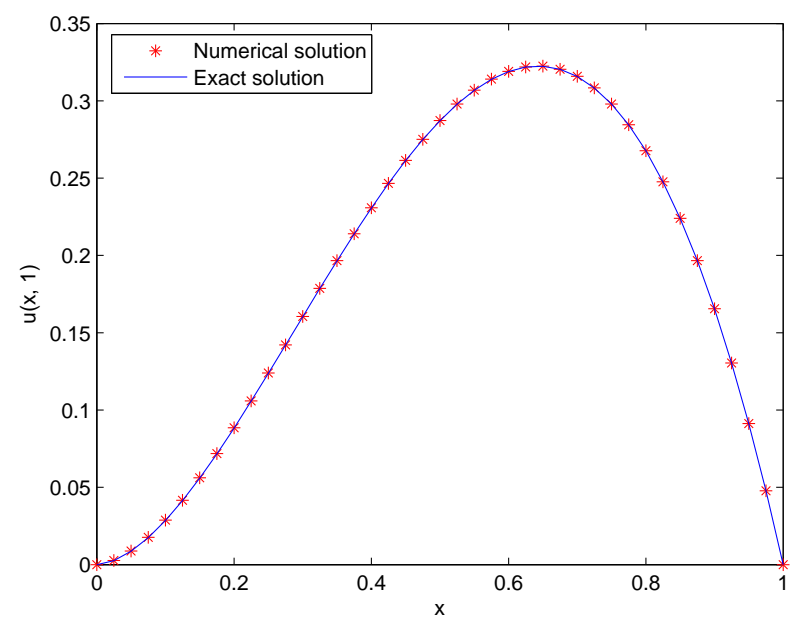

Figure 4: The numerical and exact solutions for $\alpha=0.8, \beta=0.7, \gamma=0.6$ in Example 4.4. 
Table 9: The solutions errors with time and space steps in Example 4.4.

\begin{tabular}{|cccc|}
\hline$h$ & $\tau=h^{2}$ & Err & Rat \\
\hline $1 / 4$ & $1 / 16$ & $2.48803 \mathrm{e}-2$ & \\
$1 / 10$ & $1 / 100$ & $6.12038 \mathrm{e}-3$ & 4.0652 \\
$1 / 20$ & $1 / 400$ & $2.00159 \mathrm{e}-3$ & 3.0578 \\
$1 / 40$ & $1 / 1600$ & $6.36046 \mathrm{e}-4$ & 3.1469 \\
$1 / 80$ & $1 / 6400$ & $1.98156 \mathrm{e}-4$ & 3.2098 \\
\hline
\end{tabular}

Remark 4.1. If dealing with the multiple time fractional diffusion equation where $S \geq$ 3 , we can also get good numerical solutions by the difference scheme (2.11). For example, considering the four-term time fractional diffusion equation, i.e., $S=3$ in Eq. (1), and there are $\alpha=0.85, \beta_{1}=0.7, \beta_{2}=0.5$ and $\beta_{3}=0.4$, and $r_{1}=1, r_{2}=2$ and $r_{3}=0.5$. We also take $u(x, t)=x^{2}(1-x)^{2}\left(1+t^{2}\right)$ as the exact solution, and the diffusion coefficient $D=1$, and the solutions errors at $t=1$ with the mesh $\tau=h^{2}$ are listed in Table 10, and the corresponding numerical solutions with different meshes and the exact solution also at $t=1$ are plotted in Fig. 5, respectively.

Table 10: The solutions errors with time and space steps for $S=3$ in Remark 4.1.

\begin{tabular}{|cccc|}
\hline$h$ & $\tau=h^{2}$ & Err & Rat \\
\hline $1 / 4$ & $1 / 16$ & $1.91180 \mathrm{e}-1$ & \\
$1 / 10$ & $1 / 100$ & $3.10298 \mathrm{e}-2$ & 6.1612 \\
$1 / 20$ & $1 / 400$ & $7.75687 \mathrm{e}-3$ & 4.0003 \\
$1 / 40$ & $1 / 1600$ & $1.93723 \mathrm{e}-3$ & 4.0041 \\
$1 / 80$ & $1 / 6400$ & $4.83833 \mathrm{e}-4$ & 4.0039 \\
\hline
\end{tabular}

By Table 10 and Fig. 5, we can see the difference scheme is also suitable for solving the four-term time fractional diffusion equation, and the solutions errors have the completely same trends as observed in the above, and the numerical solutions give good approximations to the exact solution with the space and time steps become small.

\section{Conclusions}

The multi-term time fractional diffusion equation can be solved analytically by Laplace transform and separating variables method using the multivariate Mittag-Leffler function. However, the solution's expression is complicated and inconvenient to utilization. The implicit finite difference scheme proposed in this paper is efficient for solving the multi-term time fractional diffusion equation numerically. The numerical solutions give good approximations to the exact solutions demonstrate the effectiveness of the proposed scheme, and the convergence rates basically coincide with the theoretical analysis as the space and time steps become small with the mesh scale of $\tau=C h^{2}$, where $\tau$ is the time step, and $h$ is the space step, $C$ is a constant. 


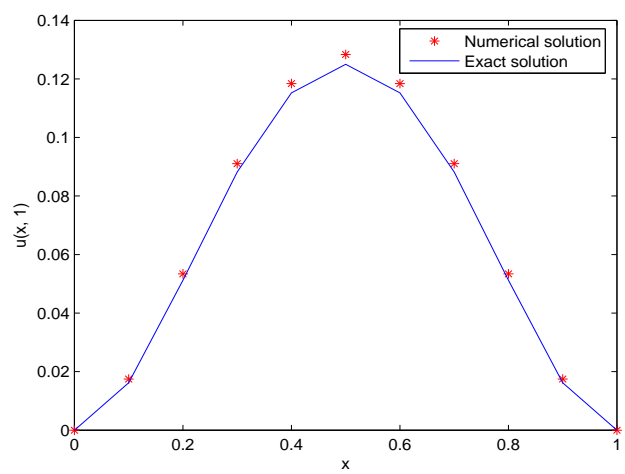

(a) $\tau=h^{2}=1 / 100$

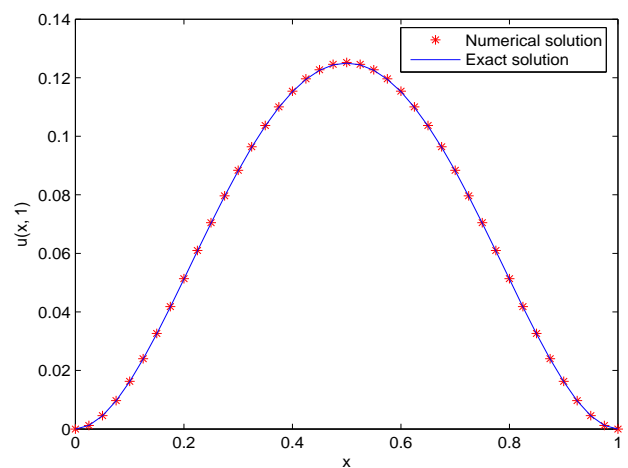

(c) $\tau=h^{2}=1 / 1600$

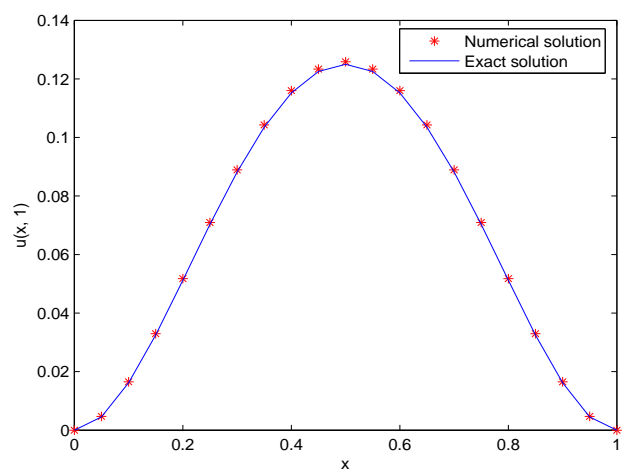

(b) $\tau=h^{2}=1 / 400$

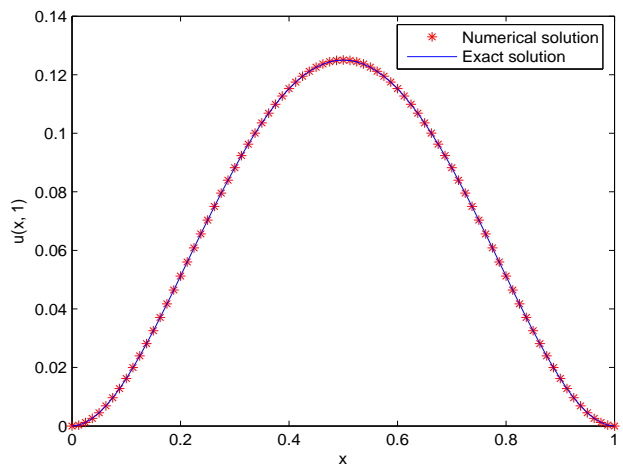

(d) $\tau=h^{2}=1 / 6400$

Figure 5: Numerical and exact solutions with different meshes for $S=3$ in Remark 4.1

The proposed method utilized in the proof of Theorems 3.2-3.3 can be generalized to establish stability and convergence of the difference scheme for other fractional differential equations, and it can also be applied to deal with numerical solutions to the multi-term time fractional diffusion equations in multidimensional cases.

Acknowledgments This work is supported by the National Natural Science Foundation of China (Grant Nos. 11071148, 11371231), and the Natural Science Foundation of Shandong Province, China (Grant No.ZR2011AQ014).

\section{Appendix A}

In this appendix, we prove the limiting expression (3.13) is valid in the proof of Theorem 3.3.

By (2.6), we have

$$
d_{n}=d_{n}^{1}+d_{n}^{2}
$$


where

$$
\begin{aligned}
d_{n}^{1} & =\frac{(n+1)^{1-\alpha}-n^{1-\alpha}}{1+\sum_{s=1}^{S} r_{s} \tau^{\alpha-\beta_{s}} \frac{\Gamma(2-\alpha)}{\Gamma\left(2-\beta_{s}\right)}} \\
d_{n}^{2} & =\frac{\sum_{s=1}^{S} r_{s}\left[(n+1)^{1-\beta_{s}}-n^{1-\beta_{s}}\right] \tau^{\alpha-\beta_{s}} \frac{\Gamma(2-\alpha)}{\Gamma\left(2-\beta_{s}\right)}}{1+\sum_{s=1}^{S} r_{s} \tau^{\alpha-\beta_{s}} \frac{\Gamma(2-\alpha)}{\Gamma\left(2-\beta_{s}\right)}}
\end{aligned}
$$

Firstly, note that

$$
\begin{aligned}
& \lim _{n \rightarrow \infty}\left[(n+1)^{1-\alpha}-n^{1-\alpha}\right] n^{\alpha}=\lim _{n \rightarrow \infty} n\left(\left(1+\frac{1}{n}\right)^{1-\alpha}-1\right) \\
= & \lim _{n \rightarrow \infty} n\left(1+\frac{1-\alpha}{n}+o\left(\frac{1}{n}\right)-1\right)=1-\alpha .
\end{aligned}
$$

Then we have

$$
\lim _{n \rightarrow \infty} d_{n}^{1} n^{\alpha}=\frac{1-\alpha}{1+\sum_{s=1}^{S} r_{s} \tau^{\alpha-\beta_{s}} \frac{\Gamma(2-\alpha)}{\Gamma\left(2-\beta_{s}\right)}} .
$$

Next, since $\tau^{\alpha-\beta_{s}}=(n \tau)^{\alpha-\beta_{s}} n^{\beta_{s}-\alpha}$, we rewrite $d_{n}^{2} n^{\alpha}$ as

$$
d_{n}^{2} n^{\alpha}=\frac{\sum_{s=1}^{S} r_{s}\left[(n+1)^{1-\beta_{s}}-n^{1-\beta_{s}}\right] n^{\beta_{s}}(n \tau)^{\alpha-\beta_{s}} \frac{\Gamma(2-\alpha)}{\Gamma\left(2-\beta_{s}\right)}}{1+\sum_{s=1}^{S} r_{s} \tau^{\alpha-\beta_{s}} \frac{\Gamma(2-\alpha)}{\Gamma\left(2-\beta_{s}\right)}}
$$

Then by $\lim _{n \rightarrow \infty}\left[(n+1)^{1-\beta_{s}}-n^{1-\beta_{s}}\right] n^{\beta_{s}}=1-\beta_{s}$, and $\lim _{n \rightarrow \infty} n \tau=T$, we can get

$$
\lim _{n \rightarrow \infty} d_{n}^{2} n^{\alpha}=\frac{\sum_{s=1}^{S} r_{s}\left(1-\beta_{s}\right) T^{\alpha-\beta_{s}} \frac{\Gamma(2-\alpha)}{\Gamma\left(2-\beta_{s}\right)}}{1+\sum_{s=1}^{S} r_{s} \tau^{\alpha-\beta_{s}} \frac{\Gamma(2-\alpha)}{\Gamma\left(2-\beta_{s}\right)}}
$$

By (A.1), and combining (A.3) with (A.4), we verified that (3.13) is valid.

\section{Appendix B}

In this appendix, we deduce the analytical solutions for the two-term and the threeterm time fractional diffusion equations given by (4.1) and (4.2) respectively. We take 
the diffusion equation with three-term time fractional derivatives as example, and consider the following initial boundary value problem

$$
\begin{aligned}
& \frac{\partial^{\alpha} u}{\partial t^{\alpha}}+r_{1} \frac{\partial^{\beta} u}{\partial t^{\beta}}+r_{2} \frac{\partial^{\gamma} u}{\partial t^{\gamma}}=D \frac{\partial^{2} u}{\partial x^{2}}, \quad 0<x<l, t>0, \\
& u(0, t)=u(l, t)=0, \quad t>0, \\
& u(x, 0)=u_{0}(x), \quad 0 \leq x \leq l .
\end{aligned}
$$

Utilizing the method of separating variables and Laplace transform, we can get the analytical solution to the problem (B.1).

Actually, noting to the homogeneous Dirichelet boundary conditions, the solution of the problem (B.1) is expressed by

$$
u(x, t)=\sum_{n=1}^{\infty} p_{n}(t) \varphi_{n}(x),
$$

where $\varphi_{n}(x)=\sqrt{2 / l} \sin \left(\frac{n \pi x}{l}\right)(n=1,2, \cdots$,$) are the orthogonal eigenfunctions corre-$ sponding to the eigenvalues $\lambda_{n}=D n^{2} \pi^{2} / l^{2}$, and $p_{n}(t)$ satisfying the fractional ordinary differential equation

$$
\frac{d^{\alpha} p_{n}}{d t^{\alpha}}+r_{1} \frac{d^{\beta} p_{n}}{d t^{\beta}}+r_{2} \frac{d^{\gamma} p_{n}}{d t^{\gamma}}+\lambda_{n} p_{n}=0
$$

with the initial condition

$$
p_{n}(0)=\left(u_{0}, \varphi_{n}\right):=p_{n}^{0} .
$$

Denoting $\bar{p}_{n}(\eta)=\int_{0}^{\infty} e^{-\eta t} p_{n}(t) d t\left(\operatorname{Re}(\eta)>\eta_{0}\right)$, and utilizing Laplace transform for equation (B.3), we have

$$
\eta^{\alpha} \bar{p}_{n}(\eta)-\eta^{\alpha-1} p_{n}^{0}+r_{1}\left(\eta^{\beta} \bar{p}_{n}(\eta)-\eta^{\beta-1} p_{n}^{0}\right)+r_{2}\left(\eta^{\gamma} \bar{p}_{n}(\eta)-\eta^{\gamma-1} p_{n}^{0}\right)+\lambda_{n} \bar{p}_{n}(\eta)=0
$$

and noting $r_{1}, r_{2}>0$ and $\lambda_{n}>0$, we can get

$$
\bar{p}_{n}(\eta)=\frac{\eta^{\alpha-1}+r_{1} \eta^{\beta-1}+r_{2} \eta^{\gamma-1}}{\eta^{\alpha}+r_{1} \eta^{\beta}+r_{2} \eta^{\gamma}+\lambda_{n}} p_{n}^{0}
$$

Denote

$$
\bar{g}_{n}(\eta)=\frac{\eta^{\alpha-1}+r_{1} \eta^{\beta-1}+r_{2} \eta^{\gamma-1}}{\eta^{\alpha}+r_{1} \eta^{\beta}+r_{2} \eta^{\gamma}+\lambda_{n}}
$$

Then it can be verified that

$$
\bar{g}_{n}(\eta)=\frac{\eta^{\alpha-\beta-1}+r_{1} \eta^{-1}+r_{2} \eta^{\gamma-\beta-1}}{\eta^{\alpha-\beta}+r_{1}} \frac{1}{1+\frac{r_{2} \eta^{\gamma-\beta}+\lambda_{n} \eta^{-\beta}}{\eta^{\alpha-\beta}+r_{1}}} .
$$

Since

$$
\frac{1}{1+\frac{r_{2} \eta^{\gamma-\beta}+\lambda_{n} \eta^{-\beta}}{\eta^{\alpha-\beta}+r_{1}}}=\sum_{m=0}^{\infty}(-1)^{m} \frac{\left(r_{2} \eta^{\gamma-\beta}+\lambda_{n} \eta^{-\beta}\right)^{m}}{\left(\eta^{\alpha-\beta}+r_{1}\right)^{m}}
$$


we have

$$
\bar{g}_{n}(\eta)=\sum_{m=0}^{\infty}(-1)^{m} \frac{\eta^{\alpha-\beta-1}+r_{1} \eta^{-1}+r_{2} \eta^{\gamma-\beta-1}}{\left(\eta^{\alpha-\beta}+r_{1}\right)^{m+1}} \sum_{k=0}^{m} C_{m}^{k}\left(r_{2} \eta^{\gamma-\beta}\right)^{k}\left(\lambda_{n} \eta^{-\beta}\right)^{m-k},
$$

where $C_{m}^{k}=\frac{m !}{k !(m-k) !}$. Then by the well-known Laplace transform formula (see, e.g., [23])

$$
\int_{0}^{\infty} e^{-\eta t} t^{\alpha m+\beta-1} E_{\alpha, \beta}^{(m)}\left(-c t^{\alpha}\right) d t=\frac{m ! \eta^{\alpha-\beta}}{\left(\eta^{\alpha}+c\right)^{m+1}}
$$

where $c$ is a constant, $m \in \mathbf{N}$, and $E_{\alpha, \beta}^{(m)}(z)=\frac{d^{k}}{d z^{k}} E_{\alpha, \beta}(z)$, and $E_{\alpha, \beta}(z)$ is the twoparameter Mittag-Leffer function defined by

$$
E_{\alpha, \beta}(z)=\sum_{j=0}^{\infty} \frac{z^{j}}{\Gamma(\alpha j+\beta)},
$$

we can get

$$
\begin{aligned}
g_{n}(t)=\sum_{m=0}^{\infty} & (-1)^{m} \lambda_{n}^{m} \sum_{k=0}^{m} \frac{\left(r_{2} / \lambda_{n}\right)^{k}}{k !(m-k) !} t^{m \alpha-k \gamma}\left(E_{\alpha-\beta, 1+m \beta-k \gamma}^{(m)}\left(-r_{1} t^{\alpha-\beta}\right)\right. \\
& +r_{1} t^{\alpha-\beta} E_{\alpha-\beta, 1+m \beta-k \gamma+\alpha-\beta}^{(m)}\left(-r_{1} t^{\alpha-\beta}\right) \\
& \left.+r_{2} t^{\alpha-\gamma} E_{\alpha-\beta, 1+m \beta-k \gamma+\alpha-\gamma}^{(m)}\left(-r_{1} t^{\alpha-\beta}\right)\right) .
\end{aligned}
$$

Hence, we get the solution of the ordinary fractional differential equation (B.3) with the initial condition (B.4) which is $p_{n}(t)=p_{n}^{0} g_{n}(t)$, and the analytical solution of problem (B.1) is given by

$$
u(x, t)=\sum_{n=1}^{\infty} p_{n}^{0} g_{n}(t) \varphi_{n}(x) .
$$

In the case of $l=\pi, D=1$, and $u_{0}(x)=\sin (x)$, there are $p_{1}^{0}=\sqrt{\frac{\pi}{2}}$ and $p_{n}^{0}=0$ for $n \geq 2$, and $\lambda_{n}=D n^{2}=n^{2}$, then the exact solution is expressed by $u(x, t)=$ $p_{1}^{0} g_{1}(t) \varphi_{1}(x)=g_{1}(t) \sin (x)$, which leads to the expression (4.6).

With a similar method as used in the above, we can get the analytical solution (4.3) for the two-term time fractional diffusion equation with the same initial boundary value conditions as used for the three-term case.

\section{References}

[1] E. E. ADAMS, L. W. GelHAR, Field study of dispersion in a heterogeneous aquifer 2: Spatial moments analysis, Water Resources Research, 28 (1992), pp. 3293-3307.

[2] D. A. Benson, The Fractional Advection-Dispersion Equation: Development and Application, Dissertation of Doctorial Degree, University of Nevada, Reno, USA, 1998.

[3] B. Berkowitz, H. Scher, S. E. Silliman, Anomalous transport in laboratory-scale heterogeneous porus media, Water Resources Research, 36 (2000), pp. 149-158. 
[4] R. Caponetto, G. Dongola, L. Fortuna, I. Petras, Fractional Order Systems: Modeling and Control Applications, World Scientific, Singapore, 2010.

[5] S. Chen, F. LiU, K. Burrage, Numerical simulation of a new two-dimensional variableorder fractional percolation equation in non-homogeneous porous media, Computers and Mathematics with Applications, 67 (2014), pp. 1673-1681.

[6] C. F. M. Coimbra, Mechanics with variable-order differential operators, Ann. Phys., 12 (2003), pp. 692-703.

[7] V. DAFTARDAR-GeJJI, S. BhaleKAR, Boundary value problems for multi-term fractional differential equationas, J. Math. Anal. Appl., 345 (2008), pp. 754-765.

[8] M. Giona, S. Gerbelli, H. E. Roman, Fractional diffusion equation and relaxation in complex viscoelastic materials, Physica A, 191 (1992), pp. 449-453.

[9] Y. Hatano, N. HATANo, Dispersive transport of ions in column experiments: an explanation of long-tailed profiles, Water Resources Research, 34 (1998), pp. 1027-1033.

[10] H. JiAng, F. LiU, I. TURner, K. BuRRAGe, Analytical solutions for the multi-term timefractional diffusion-wave/diffusion equations in a finite domain, Computers and Mathematics with Applications, 64 (2012), pp. 3377-3388.

[11] A. A. Kilbas, H. M. SRivastava, J. J. Trujillo, Theory and Applications of Fractional Differential Equations, Elsevier, Amsterdam, 2006.

[12] G. S. LI, W. J. GU, X. Z. JIA, Numerical inversions for space-dependent diffusion coefficient in the time fractional diffusion equation, Journal of Inverse and Ill-Posed Problems, 20 (2012), pp. 339-366.

[13] F. Liu, P. Zhuang, V. Anh, I. Turner, K. Burrage, Stability and convergence of the difference methods for the space-time fractional advection-diffusion equation, Applied Mathematics and Computation, 191 (2007), pp. 12-20.

[14] F. Liu, P. ZhUANG, K. BuRRAge, Numerical methods and analysis for a class of fractional advection-dispersion models, Computers and Mathematics with Applications, 64 (2012), pp. 2990-3007.

[15] F. Liu, M. M. Meerschaert, R. J. McGough, P. Zhuang, Q. Liu, Numerical methods for solving the multi-term time-fractional wave-diffusion equation, Fractional Calculus and Applied Analysis, 16 (2013), pp. 9-25.

[16] C. F. LORENZO, T. T. HARTLEY, Variable order and distributed order fractional operators, Nonlinear Dynam., 29 (2002), pp. 57-98.

[17] Y. Luснко, Maximum principle for the generalized time-fractional diffusion equation, J. Math. Anal. Appl., 351 (2009), pp. 218-223.

[18] Y. Luchко, Some uniqueness and existence results for the initial-boundary-value problems for the generalized time-fractional diffusion equation, Computers and Mathematics with Applications, 59 (2010), pp. 1766-1772.

[19] Y. LUCHKO, Initial-boundary-value problems for the generalized multi-term time-fractional diffusion equation, J. Math. Anal. Appl., 374 (2011), pp. 538-548.

[20] F. MAinARDi, Fractional Calculus and Waves in Linear Viscoelasticity: An Introduction to Mathematical Models, Imperial College Press, London, 2010.

[21] M. M. Meerschaert, C. TADJeran, Finite difference approximations for fractional advection-dispersion flow equations, Journal of Computional and Applied Mathematics, 172 (2004), pp. 65-77.

[22] H. T. C. Pedro, M. H. Kobayashi, J. M. C. Pereira, C. F. M. Coimbra, Variable order modelling of diffusive-convective effects on the oscillatory flow past a sphere, J. Vib. Control, 14 (2008), pp. 1659-1672.

[23] I. PodlubNy, Fractional Differential Equations, Academic Press, San Diego, 1999. 
[24] K. SАкамото, M. ҮАмамото, Initial value/boundary value problems for fractional diffusion-wave equations and applications to some inverse problems, J. Math. Anal. Appl., 382 (2011), pp. 426-447.

[25] L. ZHOU, H. M. Selim, Application of the fractional advection-dispersion equations in porous media, Soil. Sci. Soc. Am. J., 67 (2003), pp. 1079-1084. 